\title{
Characterisation of the Humidity and Temperature Responses of a Reflection Hologram Recorded in Acrylamide-based Photopolymer
}

\author{
Izabela Naydenova \\ Technological University Dublin, izabela.naydenova@tudublin.ie \\ Raghavendra Jallapuram \\ Technological University of Dublin, mrutic@gmail.com \\ Vincent Toal \\ Technological University of Dublin, vincent.toal@tudublin.ie
}

See next page for additional authors

Follow this and additional works at: https://arrow.tudublin.ie/cieoart

Part of the Optics Commons

\section{Recommended Citation}

Naydenova, I. et al. (2009) Characterisation of the Humidity and Temperature Responses of a Reflection Hologram Recorded in Acrylamide-Based Photopolymer. Sensors and Actuators B: Chemical, Vol. 139,no. 1, pp.35-38, May. doi: 10.1016/j.snb.2008.08.020

This Article is brought to you for free and open access by the Centre for Industrial and Engineering Optics at ARROW@TU Dublin. It has been accepted for inclusion in Articles by an authorized administrator of ARROW@TU Dublin. For more information, please contact arrow.admin@tudublin.ie, aisling.coyne@tudublin.ie, gerard.connolly@tudublin.ie.

Funder: Enterprise Ireland Technology Commercialisation Fund.

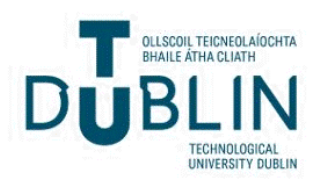




\section{Authors}

Izabela Naydenova, Raghavendra Jallapuram, Vincent Toal, and Suzanne Martin 


\title{
Characterisation of the humidity and temperature
}

\section{responses of a reflection hologram recorded in}

\author{
acrylamide-based photopolymer
}

\author{
Izabela Naydenova, ${ }^{1 *}$ Raghavendra Jallapuram, ${ }^{1}$ Vincent Toal $^{1,2}$ and Suzanne \\ $\operatorname{Martin}^{1}$ \\ ${ }^{1}$ Centre for Industrial and Engineering Optics, Dublin Institute of Technology, Kevin \\ Street, Dublin 8, Ireland \\ ${ }^{2}$ School of Physics, Dublin Institute of Technology, Kevin Street, Dublin 8, Ireland \\ *Corresponding author: izabela.naydenova@dit.ie
}

The humidity and temperature responses of a reflection hologram recorded in a selfprocessing photopolymer layer have been characterised by measurement of the position of the maximum intensity in the spectral response of the hologram. It has been demonstrated that such a hologram can be used for indication of the relative humidity $(\mathrm{RH})$ in a specific temperature range. The operational range and reversibility of the holograms have been characterised in a controlled humidity and temperature environment. The humidity studies were carried out in the range from 10 to $80 \% \mathrm{RH}$ and the temperature range under study was $15-50{ }^{\circ} \mathrm{C}$. 


\section{Introduction}

Novel sensors utilising a hologram recorded in an analyte sensitive medium have been attracting increasing interest in recent years [1-4]. The operation of such sensors is based on the fact that when the properties of the holographic recording medium are changed due to the presence of an analyte the optical properties of the recorded hologram also change. This usually leads to a visual change in the recorded holographic image that can be easily detected simply by looking at the sensor. One example is a change of the colour of the holographic image when the reflection hologram is exposed to the analyte. One further step in the development is a holographic sensor that contains more than one hologram. In this case the exposure to the analyte can lead to the disappearance of one holographic image and appearance of another [5]. The main advantages of the holographic sensors are the possibility to provide visual information easily interpreted by a non specialist, their relatively low price, flexible design and small format. For instance they can be used as smart labels in the packaging industry to monitor the content and the environment in the package. One important aim is to be able to monitor and control the humidity in packages especially when used to transport electronic goods. At present the humidity in electronic packaging is monitored with the help of a card containing spots of silica gel that indicate a change in relative humidity by a colour change. However, such devices have a slow response time, limited accuracy and can not be used for authentication of the goods origin. Holograms capable of indicating the humidity can prove very useful in combining sensing and security features.

Another application of humidity sensitive holograms could be to indicate the environmental humidity. Usually it is the RH rather than the absolute humidity that is 
of interest in this case. At present relative humidity is measured by detecting the change of a physical property of a thin sensing film such as capacitance, resistivity, thermal conductivity, etc in response to the change in the relative humidity of the environment. When used in humidity control systems such humidity sensors are characterised by high accuracy in the order of a fraction of a percent. However, such devices need a power source; they are relatively complex, expensive and bulky devices which include electronic components. Holographic sensors can find application where cheap, disposable relative humidity indicators, providing information at a glance without additional power source are required. We have recently reported some initial results on the design and fabrication of a humidity sensitive reflection hologram that could be used in visual indication of the environmental humidity [4]. Here we present results from further characterisation of its temperature and humidity response. These results will be useful to determine the humidity and temperature ranges in which these devices can be used for RH indication.

\section{Theory}

The recording of a hologram requires two beams of coherent light, one object and one reference beam and a photosensitive medium located in the region where the two beams intersect and produce an interference pattern. This interference pattern is then recorded as a change in the optical properties (refractive index and/or absorption coefficient) of the recording medium and thus the hologram containing full information about the phase and the amplitude of the object beam is created. When, at a later stage, the hologram is illuminated with the reference beam, the object beam is reconstructed. In the simplest example when the two beams are plain waves incident 
from the two opposite sides of the recording medium, the hologram is a reflection grating with a period $\Lambda$, determined by:

$$
\Lambda=\frac{\lambda}{2 n \sin \theta}
$$

where $n$ is the average refractive index of the recording medium, $\lambda$ is the recording wavelength and $\theta$ is the half of the angle between the two recording beams. When such a holographic grating is illuminated with white light the diffracted light in a specific direction will be of wavelength $\lambda$ given by eq. (1) and a single colour will be observed. In order to analyse what could be the reasons for change of the colour/wavelength of the diffracted light one can differentiate equation (1):

$$
\Delta \lambda=2 \sin \theta(n \Delta \Lambda+\Lambda \Delta n)+2 n \Lambda \cos \theta \Delta \theta
$$

It is seen from equation (2) that a change of the wavelength of the diffracted light could be caused by change of the average refractive index, change in the grating period or when observing at different angle. Since in reflection mode of recording the angle between the two recording beams $(2 \theta)$ is usually close to $180^{\circ}$ the second term in equation (2) could be negligibly small. In this case the change in the diffracted wavelength would be due to the first two factors. Both of them - change in the average refractive index, and change in the grating period could be caused by the presence of the analyte. In [4] we have estimated the relative contribution of the two factors in the case of a humidity sensitive reflection hologram recorded in acrylamide based photopolymer layer and have concluded that the main factor that could lead to diffracted light wavelength shift of more than $100 \mathrm{~nm}$ is the change of the grating period. The grating period is expected to change if a hologram experiences change of 
its dimensions as shown in Fig. 1. The photopolymer layers described in [4] and in this paper contain a significant amount of PVA and polyacrylamide, both highly hygroscopic materials. When exposed to high humidity the hologram absorbs water and swells, resulting in a red shift in the diffracted light wavelength. When the hologram is exposed to low humidity the water is released, the hologram shrinks and a blue shift in the diffracted light wavelength is observed. The experiments reported in [4] were carried out at constant temperature. For practical applications of these humidity sensitive holograms it was important to characterise also their response at different temperatures. The results are presented in the following sections.

\section{Experimental}

The holographic recording material used in this study is a self-processing acrylamide based photopolymer developed at the Centre for Industrial and Engineering Optics, Dublin Institute of Technology [6-8]. The photosensitive layer consists of two monomers - acrylamide and N,N Methylene bisacrylamide, a polymeric binder, polyvinyl alcohol, a photosensitiser, Erythrosin B and a co-initiator, triethanolamine (TEA). The optimised composition for recording reflection holograms contains $0.8 \mathrm{~g}$ acrylamide, $0.25 \mathrm{~g} \mathrm{~N}, \mathrm{~N}$ 'methylene bisacrylamide, $1.5 \mathrm{ml}$ triethanolamine, $17.5 \mathrm{ml}$ of $10 \% \mathrm{w} / \mathrm{v}$ polyvinylalcohol stock solution and $3 \mathrm{ml}$ of $0.11 \% \mathrm{w} / \mathrm{v}$ of Erythrosin B stock solution. Layers of thickness $30-180 \mu \mathrm{m}$ were obtained by varying the volume of the photopolymer solution gravity settled on a $50 \mathrm{x}$ $50 \mathrm{~mm}^{2}$ glass slide between 0.5 and $2 \mathrm{ml}$. The samples were dried for 24 hours.

The thickness of the dry photopolymer layer was measured by cutting the photopolymer layer using a sharp scalpel and measuring the dimensions of the cut with a white light interferometric (WLI) surface profiler MicroXAM S/N 8038. 
Reflection gratings were recorded using the recording set up shown in Fig.2. The frequency doubled output from $\mathrm{NdYVO}_{4}$ laser (Verdi 05) was used to record the holograms at a wavelength of $\lambda=532 \mathrm{~nm}$. The recording intensities of the two recording beams B1 and B2 were adjusted with the help of a polarising beam splitter (PBS) and two half wave plates (HW).

A controlled environment chamber with humidity and temperature control system was purchased from Electro-tech systems, Inc. The system, model 5503-11, consists of a glove box, a microprocessor controller, a dehumidification system, an ultrasonic humidification system and thermoelectric cooling and heating systems. The $\mathrm{RH}$ in the chamber can be maintained to better than $\pm 1 \% \mathrm{RH}$ of the set point. The operational range is from $5 \%$ to $100 \% \mathrm{RH}$. A fast responding capacitive type sensor providing measurement accuracy of $\pm 2 \% \mathrm{RH}$ over the range of $0-90 \%$ and $3 \%$ from 90 to $100 \% \mathrm{RH}$ is incorporated in the chamber. The temperature in the chamber is maintained within $0.1{ }^{\circ} \mathrm{C}$ of the set point. The optical set-up for measuring the spectral characteristics of the light diffracted from the hologram was assembled in the humidity chamber (Fig.3). The probe light from a broad band light source (AvaLightHAL-S) was fiber guided into the humidity chamber. The diffracted light was then coupled into a second fiber by a lens and guided to a spectral analyzer, AvaSpec2048. In order to obtain the humidity or temperature response of a specific hologram the relationship between the peak wavelength of the light diffracted from the hologram and the RH or the temperature respectively was determined.

\section{Results and Discussion}

The humidity response of a $30 \mu \mathrm{m}$ thick reflection hologram, recorded at $40 \% \mathrm{RH}$ using total intensity of $6 \mathrm{~mW} / \mathrm{cm}^{2}$ and recording time of $40 \mathrm{~s}$ are presented in Fig. 4. 
The spatial frequency of the hologram was 5580 lines $\mathrm{mm}^{-1}$, corresponding to a grating period of approximately $180 \mathrm{~nm}$. The temperature in this experiment was kept constant at $23^{\circ}$ C. In Fig. 4 the spectral distribution of the diffracted light intensity, also known as the wavelength selectivity or Bragg curve is presented. It is seen that with an increase of the humidity in the environmental chamber the Bragg curves shift towards higher wavelengths. Figure $4 \mathrm{~b}$ depicts the relation between the peak wavelength of the curves presented in Fig. 4 a and the RH. One can estimate that a change in the RH from 15 to $65 \%$ leads to a shift in the peak wavelength of more than $80 \mathrm{~nm}$. This corresponds to approximately $1.3 \mathrm{~nm}$ per $1 \%$ change in the $\mathrm{RH}$. Taking into account that the wavelength resolution limit of an average human eye is 2 $\mathrm{nm}$, one can estimate that a change in the $\mathrm{RH}$ of $5 \%$ can be easily monitored by simply glancing at the hologram. It is also worth noting that the sensitivities of the hologram are different at the low and high RH ranges. For instance a change of $10 \%$ RH from 55 to $65 \%$ leads to $28 \mathrm{~nm}$ shift while the same $10 \%$ change from 15 to 25 $\% \mathrm{RH}$ in this sample leads to only $1 \mathrm{~nm}$ shift. It is clear that, if the hologram is to be used to monitor packages requiring low humidity, better response must be sought. This could be achieved by variation of the recording material chemical composition and also perhaps by optimising the recording geometry. In order to optimise the sensor for a specific operational range it is important to understand the mechanism behind the changes caused by the analyte. If one takes into account the extent of the wavelength shift and one assumes that only one of the first two terms in equation (2) can contribute to this shift, one can estimate that it could be caused either by change in the grating period, $\Delta \Lambda$, of $27 \mathrm{~nm}$ or by a change in the refractive index $\Delta \mathrm{n}$ of 0.22 . While a change in the refractive index of 0.22 seems unrealistically large, the change of the grating period constitutes $15 \%$ of the fringe spacing and a change of this 
magnitude can be envisaged, taking into account the hydrophilic nature of the PVA and the polyacrylamide which are the main components in the photopolymer layer.

In order to further check this hypothesis, gravimetric studies were carried out in addition to the studies of the spectral response at different humilities and constant temperature of $23^{\circ} \mathrm{C}$. The sample consisting of the hologram deposited on a glass substrate was weighed at different relative humidity levels ranging from $15 \% \mathrm{RH}$ to $65 \% \mathrm{RH}$. The relation between the peak wavelength position change and the mass change is shown in Fig. 4c. As seen from the figure the relationship between these two quantities is to a good approximation linear, as would be expected if the main contributor to the change in spectral response originates from the dimensional changes in the layer. One can also estimate the expected slope of the linear dependence of the wavelength change $\Delta \lambda$ and the absorbed amount of water $\Delta m$ using the equation (3)

$$
\Delta \lambda=2 n \Delta \Lambda=\frac{2 \cdot n \cdot \Lambda \cdot \Delta d}{d}=\frac{2 \cdot n \cdot \Lambda \cdot \Delta V}{d \cdot S}=\frac{2 \cdot n \cdot \Lambda \cdot \Delta m}{d \cdot S \cdot \rho}
$$

where $\mathrm{n}$ is the refractive index of the photopolymer $(\mathrm{n}=1.5), \Lambda$ is the fringe spacing (for the grating under study $\Lambda=180 \mathrm{~nm}$ ), $d$ is the thickness of the layer ( $d=30 \mu \mathrm{m}$ ) and $S$ is the area of the photopolymer film $\left(2.6 \times 2.6 \mathrm{~cm}^{2}\right), \rho$ is the density of water $\left(0.99756\right.$ at $\left.23{ }^{\circ} \mathrm{C}\right)$. After linear fit of the measured experimental dependence $\Delta \lambda(\Delta \mathrm{m})$ it was found out that the slope is $10.5 \mathrm{~nm} / \mathrm{mg}$. The theoretically predicted slope, using equation (3) was estimated to be $26.7 \mathrm{~nm} / \mathrm{mg}$. One possible reason for this lower sensitivity of the hologram could be the fact that the polyacrylamide is a highly porous material and that some of the water is absorbed without causing dimensional change in the photopolymer layer. This is most probably the reason for the very low sensitivity at low relative humidity. 
The temperature response at four different RH levels - 15\%, 30\% and $45 \%$ and 60 $\%$ is presented in fig.5. It is seen from the figure that up to $30 \% \mathrm{RH}$ there is no temperature dependence of the spectral response of the hologram. Taking into account that:

$$
R H[\%]=\frac{m_{\text {present }}}{m_{\text {saturated }}} \times 100,
$$

where $m_{\text {present }}$ is the mass of the water vapour present in the air and $m_{\text {saturated }}$ is the mass of the vapour required to saturate the air at the same temperature one can calculate that when the $\mathrm{RH}$ is kept constant and the temperature changes from $15^{\circ} \mathrm{C}$ to $35^{\circ} \mathrm{C}$ the present amount of water in the humidity chamber increases more than three times. Nevertheless, little or no change is observed in the response of the reflection grating at $\mathrm{RH}$ up to $45 \%$. It was observed that if the $\mathrm{RH}$ is increased above this value the temperature dependence becomes pronounced even in the lower end of the measured temperature range when the temperature changes from $15^{\circ} \mathrm{C}$ to $30^{\circ} \mathrm{C}$. More detailed measurements of the temperature dependence at higher $\mathrm{RH}$ values are in progress.

In order to check the temporal stability of the hologram, the sample was left at RH $15 \%$ and the maximum achievable temperature in this chamber of $50{ }^{\circ} \mathrm{C}$ for 12 hours. No change of the position of the peak wavelength of the diffracted light was observed. The sample was also exposed to cyclic change of RH from 20 to $80 \%$. The change in the two wavelength peak positions, at 20 and at $80 \% \mathrm{RH}$ was repeatable within $2 \mathrm{~nm}$ of their original values.

\section{Conclusions}


In summary we have characterised the humidity and the temperature response of a reflection hologram recorded in an acrylamide - based photopolymer layer, when RH varies from 10 to $70 \%$ and the temperature varies from 15 to $50{ }^{\circ} \mathrm{C}$. The results reveal that if the temperature is kept constant within $5{ }^{\circ} \mathrm{C}$ the hologram can be used as a reversible RH indicator. Different calibration curves might be required at different temperatures. At temperatures from 15 to $30{ }^{\circ} \mathrm{C}$ and $\mathrm{RH}$ below $45 \%$ the hologram can be used as $\mathrm{RH}$ indicator using only one calibration curve. Above $30{ }^{\circ} \mathrm{C}$ a temperature correction of the calibration curve would be required. If the working $\mathrm{RH}$ range is restricted to $30 \%$ the temperature range can be extended to at least $50{ }^{\circ} \mathrm{C}$. The hologram could prove useful for application in monitoring the $\mathrm{RH}$ in packaging for electronics where low $\mathrm{RH}$ is important. Further optimisation is required to improve the sensitivity of the hologram in this RH range in order to obtain working devices.

\section{Acknowledgments}

The authors would like to acknowledge the financial support from Enterprise Ireland through the Commercialisation Fund, Technology Development phase.

\section{References}

[1] A.J. Marshall, J. Blyth, C. Davidson, C.R. Lowe, pH-Sensitive Holographic Sensors, Analyt. Chem. 75, (2003) 4423- 4431.

[2] C.R.Lowe, P. James, E. Rayne, S. Kabilan, J. Marshall, Holographic sensor having heterogeneous properties, WO2006008524.

[3] I. Naydenova, R. Jallapuram, V. Toal, S. Martin, Hologram-based humidity indicator for domestic and packaging applications, SPIE proceedings, 6528, (2007) 652811. 
[4] I. Naydenova, R. Jallapuram, V. Toal, S. Martin, A visual indication of environmental humidity using a colour changing hologram recorded in a selfdeveloping photopolymer, Appl. Phys. Lett. 92 (2008) 031109.

[5] M. R. Bradley, Sensor with holographic multiplexed image display, WO0150113.

[6] S. Martin, P. Leclere, Y. Renotte, V. Toal, Y. Lion, Characterisation of an acrylamide-based dry photopolymer holographic recording material, Opt. Eng. 33 (1992) 3942-3946..

[7] S. Martin, C. Feely, V. Toal, Holographic recording characteristics of an acrylamide-based photopolymer, Appl. Opt. 36 (1997) 5757-5768.

[8] I. Naydenova, S. Martin, R. Jallapuram, R. Howard, V. Toal, Investigations of the diffusion processes in self-processing acrylamide-based photopolymer system, Appl. Opt. 43 (14) (2004) 2900-2905. 
Figure captions:

Fig. 1 Principle of operation of the humidity/temperature sensitive hologram. A dimensional change, shrinkage or swelling of the photopolymer layer, leads to a change of the fringe spacing and a change of the wavelength of the diffracted light in a specific direction.

Fig. 2 Recording set-up: S-electronic shutter; HW - halfwave plate; PBS - polarising beam splitter; SF - spatial filter; CL - collimator; M - mirror; PL - photopolymer layer; B1, B2 - recording beams; $\mathrm{B} 1_{\mathrm{R}}, \mathrm{B} 2_{\mathrm{R}^{-}}$Fresnel reflections

Fig. 3 Test set-up: HTCE - humidity and temperature controlled environment; Hhologram; 3D - 3D translational stage; CL - collimating lens; OF - optical fibre; WLS - white light source; SA - spectrum analyser.

Fig.4 a) Wavelength Bragg selectivity curves - solid line - RH $15 \%$, dash - RH $30 \%$, dot - RH $45 \%$, dash dot - RH $57 \%$, dash dot dot - RH 60\%; b) Humidity response at constant temperature of $23^{\circ} \mathrm{C}$; c) Dependence of the change in the spectral peak position on the change in the water content in the photopolymer layer.

Fig. 5 Temperature response at constant humidity of $15 \%$ (black circles), $30 \%$ (grey triangles), $45 \%$ (light grey triangles) and 60\% (black diamonds). 


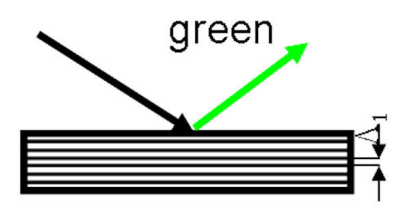

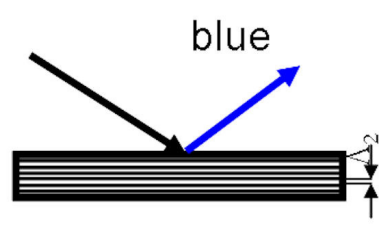

Shrinkage

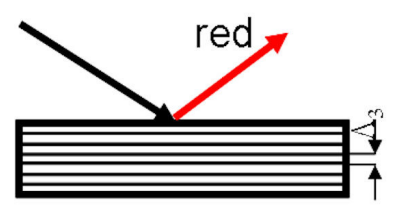

Swelling

Fig.1

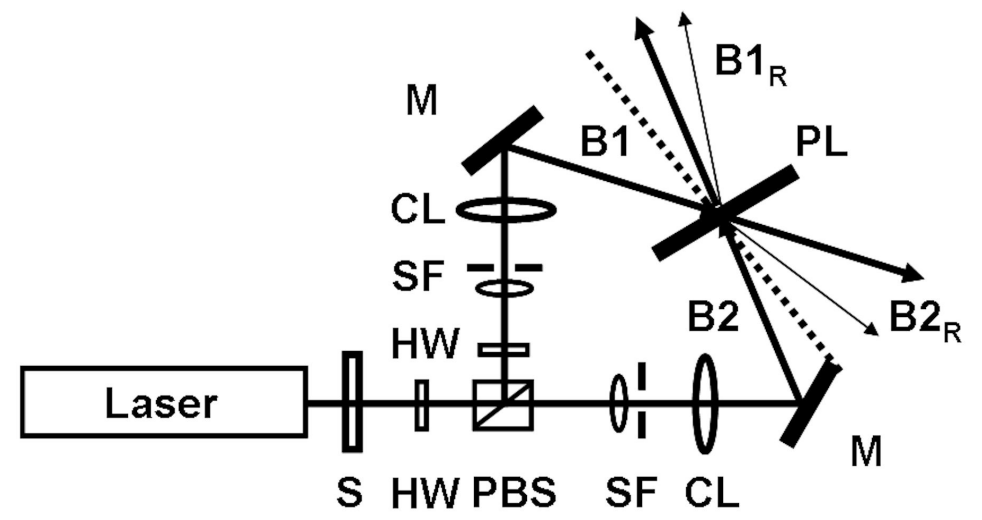

Fig. 2

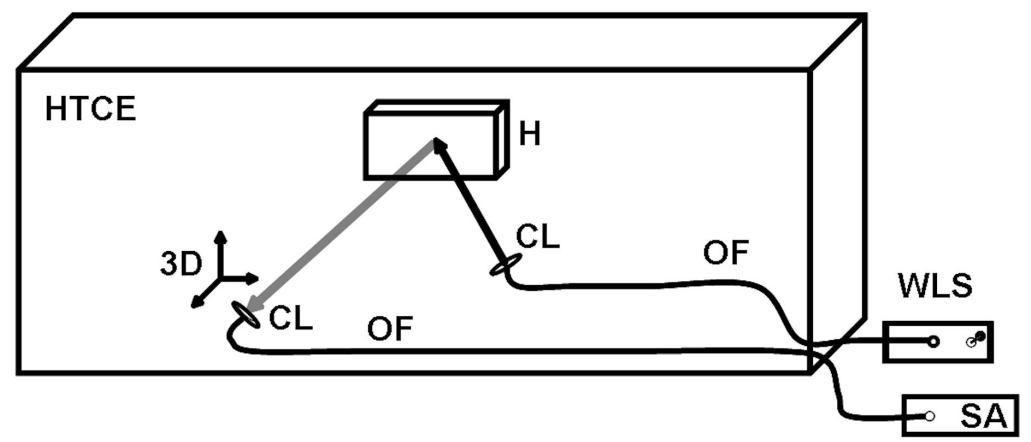

Fig. 3 


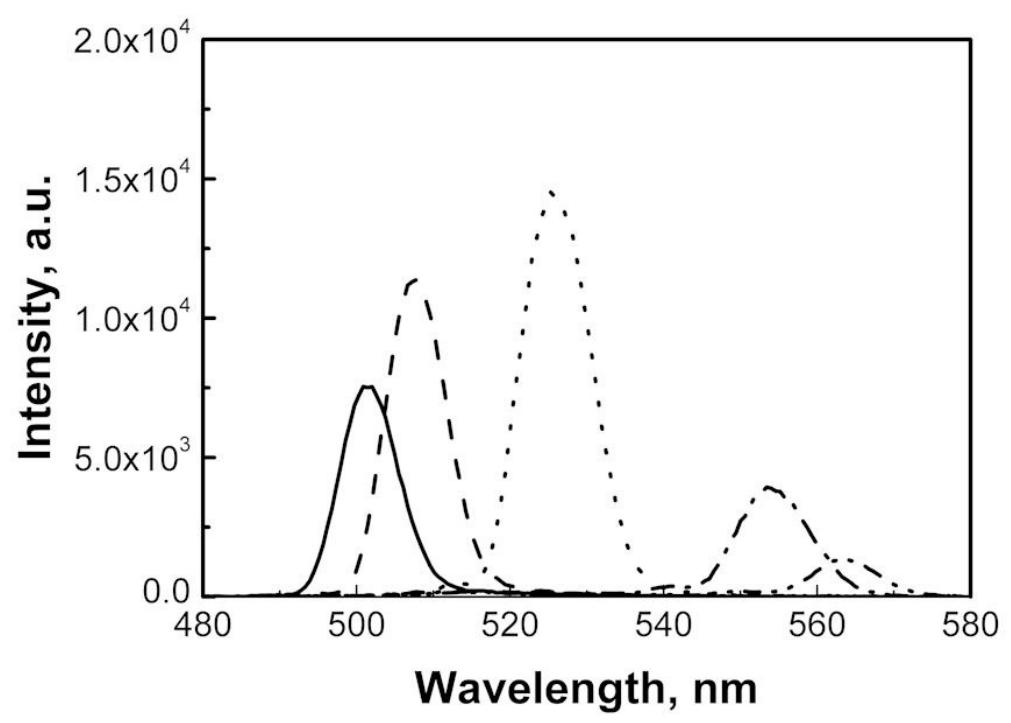

Fig.4a

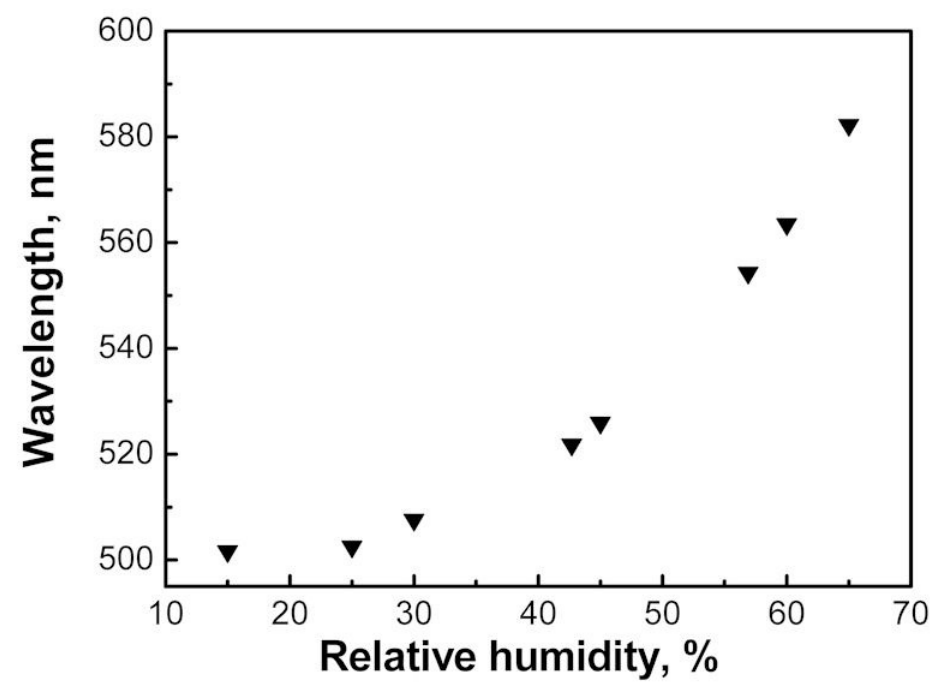

Fig.4b 


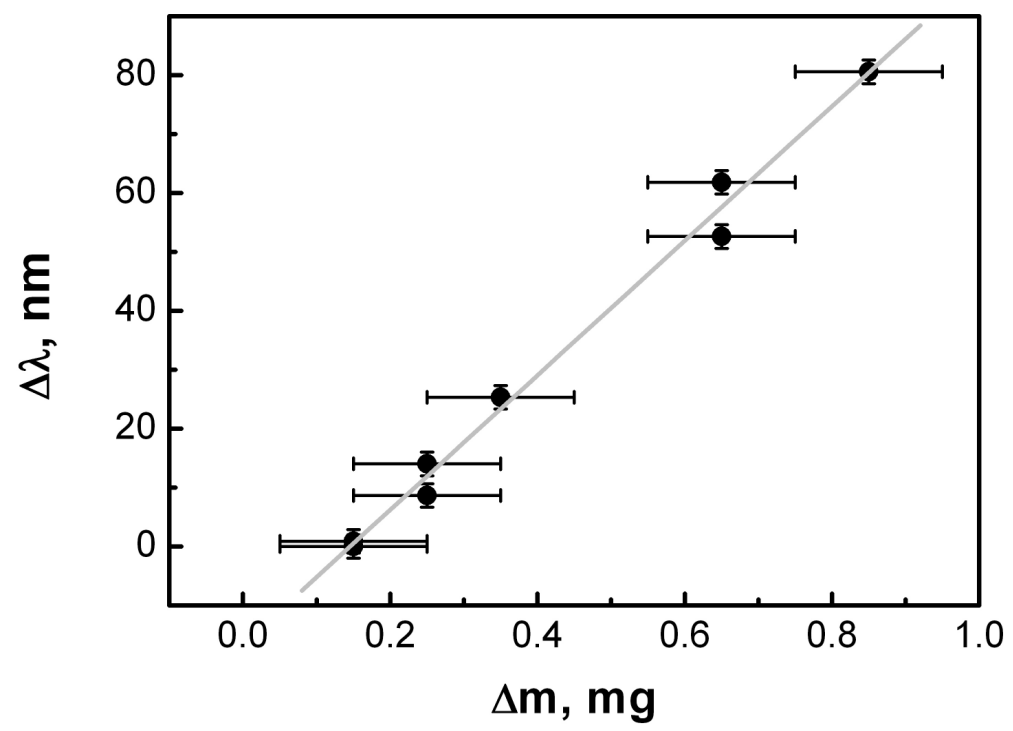

Fig.4c

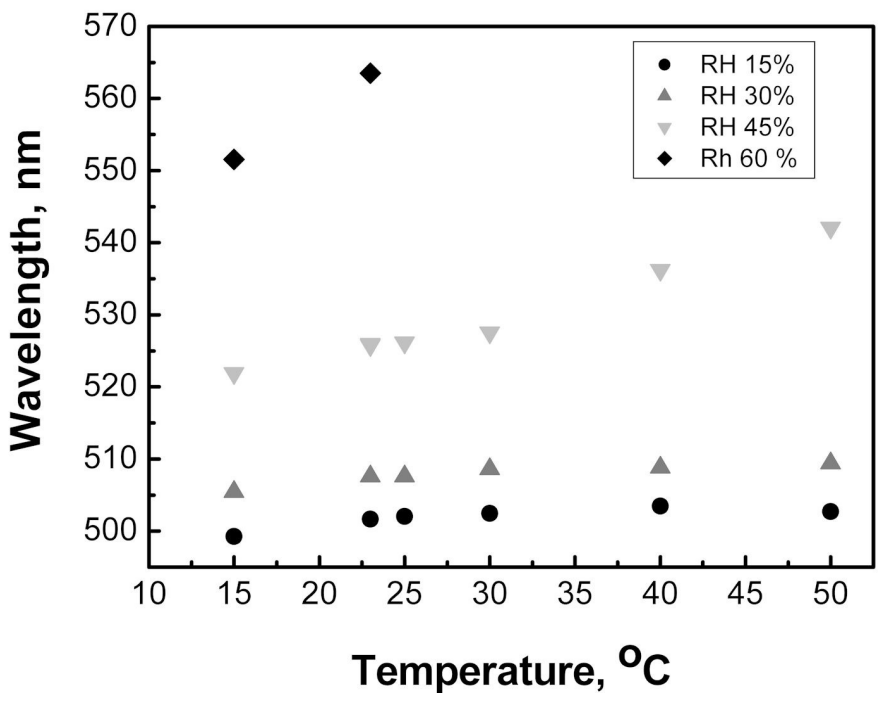

Fig.5 